\title{
Morbidity and mortality in medium chain acyl coenzyme A dehydrogenase deficiency
}

\author{
Bridget Wilcken, Judith Hammond, Martin Silink
}

\begin{abstract}
Medium chain acyl coenzyme A dehydrogenase (MCAD) deficiency presents with episodic fasting, hypoketotic hypoglycaemia, and coma. It is known to be potentially lethal, but the outlook for survivors is thought to be good. We reassessed all patients with MCAD deficiency diagnosed in New South Wales (population six million) to explore long term morbidity and mortality.

There were 16 probands and two siblings were confirmed and two presumed to be affected. Assuming an incidence of $1: 20000$ births, these represented about $22 \%$ of the total number of expected cases. Five $(25 \%)$ of the 20 patients died aged 3 days -30 months, all during the first episode of illness. Seven others had only one episode and one affected sibling was asymptomatic. Eight had had significant neonatal symptoms. Only two had a significant, serious life threatening episode after diagnosis. Of 15 survivors, one has severe handicap after a single severe episode, and four, aged 9-17 years, have mild intellectual handicap. Eight (including six aged less than 7 years), have apparently normal development. Two are lost to follow up.

Our study of unselected patients with MCAD deficiency from a defined population shows not only a substantial risk of death, but also of long term morbidity.
\end{abstract}

(Arch Dis Child 1994; 70: 410-412)

Medium chain acyl coenzyme A dehydrogenase (MCAD) deficiency is the most frequently diagnosed disorder of fatty acid $\beta$ oxidation, clinically characterised by episodes of fasting coma associated with hypoketotic hypoglycaemia and hepatic dysfunction, and often precipitated by intercurrent illness. Between episodes, patients are asymptomatic. ${ }^{1}$

The diagnosis of MCAD deficiency has become easier in recent years. There is a typical organic aciduria, even in patients who are asymptomatic, with increased levels of acylglycines, and there is a common DNA mutation, an $A$ to $G$ transition at position 985 in the coding region, which occurs in about $90 \%$ of MCAD genes, with $99 \%$ of patients with MCAD deficiency carrying at least one copy of the mutation. A specific loading test has also been described. ${ }^{2}$

It is well known that MCAD deficiency is potentially lethal, ${ }^{1}$ but the outlook for survivors has generally been assumed to be good. ${ }^{3}$ To investigate further the natural history of MCAD deficiency in a defined population, we reassessed all patients with the disease who had ever been diagnosed in New South Wales and the Australian Capital Territory (present population $6 \cdot 1$ million, birth rate about 92 000/year).

\section{Patients and methods}

PATIENTS

The patients were 16 propositi, two affected siblings, and two siblings presumed to be affected. The latter had died after a vomiting illness associated with progressive neurological obtundation, features suggestive of MCAD deficiency. The patients had been diagnosed between 1976 and 1993, either as having MCAD deficiency or as suspected cases of fatty acid oxidation defect; the diagnosis has subsequently been confirmed (except in the two patients with presumed MCAD deficiency) by the analysis of stored urine samples by gas chromatography-mass spectrometry for the presence of increased concentrations of acyl glycines (14 patients), by phenylpropionic acid loading tests (five patients), and by analysis of blood spot DNA for the A to $G$ transition at position 985 (15 families).

Management after diagnosis was confined to advice about the avoidance of fasting. No child was treated with carnitine. Only the youngest patient was treated with riboflavin $(12.5 \mathrm{mg} /$ day $)$. There were 23 siblings of the index patients. Four were affected or presumed to be affected, and 13 others were investigated and were not affected. Six declined investigation.

\section{METHODS}

All case notes were reviewed and 14 of the 16 parents were interviewed by telephone or in the clinic. Unfortunately, the parents of two patients could not be contacted. Ten patients had been seen in clinic within the preceding 12 months.

Trimethylsilyl derivatives of urinary organic acids were determined or redetermined using a Hewlett-Packard 5971 chromatograph-mass spectrometer. Phenylpropionic acid loading tests were conducted according to the method of Seakins and Rumsby. ${ }^{2}$ The A to $G$ transition at position 985 of the coding region of the MCAD gene was sought by polymerase chain reaction methodology in filter paper blood samples: 


\section{Results}

MORTALITY

Five of the 20 patients had died (including the two patients with presumed MCAD deficiency). Ages at death were 3 days $^{4}$ and 7, 10,24 , and 30 months. Each patient died during the only recognised episode of illness. Only the infant dying at 3 days had showed any symptoms in the neonatal period. In the two youngest patients, death was associated with low output cardiac failure. The older three had all had a vomiting illness for several days before death.

\section{AGE AT FIRST EPISODE}

Eight of the 20 patients had had significant hypoglycaemia requiring at least intravenous glucose at an age of 1 to 3 days. Other symptoms included hypotonia (seven patients), lethargy (six patients), vomiting (three patients), and cardiac problems (two patients).$^{5}$ Excluding neonatal symptoms, the first episodes were experienced at 5-30 months. Five children had symptoms in the neonatal period only.

\section{NUMBERS OF EPISODES}

At the time of diagnosis one patient, detected by family studies, had had no symptoms, 13 (including the five who died) had had no previous episodes of illness, three had had one previous episode, one had had two previous episodes, and one had had at least three episodes before that leading to diagnosis. After diagnosis only two patients had episodes requiring admission to hospital and intravenous fluids, and one had had four such episodes. Several other patients had had brief admissions to hospital but did not require intravenous fluids, and these episodes were more in the nature of false alarms.

\section{OUTCOME IN SURVIVORS}

Of the 15 survivors, one had severe handicap including epilepsy, hemiplegia, and marked delay with no speech after a single severe episode during which he probably was hypoglycaemic for six hours. Four patients had failed at school but one of these was a boy with an XXY karyotype (Klinefelter's syndrome), eight were making normal progress, and two were lost to follow up. Of the patients now older than 7 years four had mild handicap and only two were making normal progress at school. The two remaining older patients could not be contacted (table). Of the patients with mild handicap, all had had formal

Outcome in 15 survivors. Values given are numbers of patients

\begin{tabular}{lllll}
\hline $\begin{array}{l}\text { Current } \\
\text { age (years) }\end{array}$ & $\begin{array}{l}\text { Normal } \\
\text { progress }\end{array}$ & $\begin{array}{l}\text { Mild } \\
\text { handicap }\end{array}$ & $\begin{array}{l}\text { Severe } \\
\text { handicap }\end{array}$ & $\begin{array}{l}\text { Lost to } \\
\text { follow up }\end{array}$ \\
\hline$<7$ & $6(4 \mathrm{~N})$ & 0 & 1 & 0 \\
$\geqslant 7$ & 2 & $4^{\star}(2 \mathrm{~N})$ & 0 & $2(1 \mathrm{~N})$
\end{tabular}

$\mathrm{N}=$ neonatal symptoms (see text)

*One boy had Klinefelter's syndrome. contact with a developmental disabilities team or with school assessment for low achieving children, and all were slower than their siblings without MCAD deficiency. No patient had any overt cardiac or skeletal muscle disorder. No definitive study was routinely carried out, however.

\section{DNA MUTATIONS IN THE 15 FAMILIES}

Thirteen propositi were homozygous for the A to $G$ transition at position 985 , two had one copy of this mutation, and in one the DNA test could not be carried out.

\section{Discussion}

This study was carried out in a defined population consisting of the whole of New South Wales and the Australian Capital Territory, and not including patients from outside these regions. Thus there is not the same potential for bias as exists in reports derived from referral centres serving ill defined populations. There was a relatively high level of awareness of fatty acid oxidation disorders in this area, and we had diagnosed probable fatty acid oxidation disorders as early as $1976 .^{6}$ Nevertheless, it is certain that we have diagnosed only a small proportion of the existing cases. Studies of heterozygote frequency ${ }^{7}$ have suggested an incidence of MCAD deficiency in our population of $1 / 15000$ to $1 / 20000$. Assuming an incidence of $1 / 20000$, in the cohort born from 1975 to 1984 we would have expected 42 cases, but diagnosed only nine (21\%). From 1985 to 1991 we would have expected 31, but diagnosed only seven $(23 \%)$. Only three patients from that cohort died, so it seems that mortality could in reality be much less than the $25 \%$ we and others have observed, ${ }^{1}$ but it was at least $4 \%$. Similarly, the rate of any handicap was at least $7 \%$.

The percentage of cases of MCAD deficiency diagnosed in New South Wales cannot be considered unusually low, but rather the reverse. In most northern European countries for which data are available, the percentage of expected cases, calculated by heterozygote frequency, which have actually been diagnosed is lower, and is probably around $5-10 \%{ }^{8}$

Our experience of MCAD deficiency differs in some respects from that published previously. ${ }^{3}$ The first recognised episode was in the neonatal period in eight of our 20 patients, though such a presentation has only been recorded in four previous patients. ${ }^{4}$ It is possible that some of the other children did have undiagnosed neonatal hypoglycaemia, as fatty acids are a favoured source of energy soon after birth.

The adverse outcomes in some of our patients may have been related to the severity of the episode(s) they had before diagnosis. Certainly the boy with severe handicap had been hypoglycaemic for a prolonged period. There was no clear cut segregation with mildly adverse outcome and neonatal hypoglycaemia (table). It is not possible to be certain about 
later intellectual outcome in very young children, however, and all but two of our patients making normal progress were less than 7 years old. There was no obvious association between outcome and genotype in this small series. The most severely affected survivor and the only asymptomatic patient were siblings.

In our series there was no mortality after the diagnosis was made, and this has been the usual but not invariable finding. ${ }^{1}$ Indeed, only two of our patients had any serious episode after diagnosis, so that early and prompt diagnosis seems to lead to the effective management of this disorder.

Although no patient showed any overt skeletal or cardiac muscle disorders or growth deficit, one of the 15 survivors has severe psychomotor delay, and an unexpectedly large proportion of the older children have mild delay or failure at school. The numbers are small, and this could be a chance finding or could be due to better current management of hypoglycaemic episodes. Long term follow up of more patients is needed before the risk of damage is known. The undiagnosed patients in our state may all be asymptomatic. It is likely that some died, however, possibly as neonates, and that others had episodes of illness from which they survived handicapped, but still undiagnosed. The natural history of MCAD deficiency is not yet clear.

The authors are grateful to Dr Sue Forrest and Michaela Balnaves of the Murdoch Institute, Melbourne, for the DNA Bainaves
analyses. 1 Roe CR, Coates PM. Acyl CoA dehydrogenase deficiencies.
In: Scriver CR, Beaudet AL, Sly WS, Valle D, eds. The metabolic basis of inherited disease. 6th Ed. New York: McGraw Hill, 1989: 889-914.

2 Seakins JWT, Rumsby G. The use of phenylpropionic acid as a loading test for medium-chain acyl-CoA dehydrogenase deficiency. F Inherited Metab Dis 1988; 11: 221-4.

3 Touma EH, Charpentier C. Medium chain acyl CoA Touma EH, Charpentier C. Medium chain acyl CoA
dehydrogenase deficiency. Arch Dis Child 1992; 67: 142-5.

4 Leung K-C, Hammond JW, Chabra S, Carpenter KH, Potter $M$, Wilcken $B$. A fatal neonatal case of medium-chain acylcoenzyme A dehydrogenase deficiency. $\mathcal{F}$ Pediatr 1992; 121: 965-8.

5 Wilcken B, Carpenter KH, Hammond JW. Neonatal symptoms in medium chain acyl-coenzyme A dehydrogenase deficiency. Arch Dis Child 1993; 69: 292-4.

6 Truscott R, Hick L, Pullin C, et al. Dicarboxylic aciduria: the response to fasting. Clin Chim Acta 1979; 94: 31-4.

7 Matsubara Y, Narisawa K, Tada K, et al. Prevalence of $\mathrm{K} 329 \mathrm{E}$ mutation in medium-chain acyl-CoA dehydrogenase gene determined from Guthrie cards. Lancet 1991; 338: $552-3$.

8 Gregersen N, Winter V, Curtis D, et al. Medium chain acylCoA dehydrogenase deficiency; the prevalent mutation G985 (K304E) is subject to a strong founder effect from northern Europe. Hum Hered 1993; 43: 342-50. 